MITOCHONDRIA

\section{Getting together}

Mitochondria are enjoying something of a renaissance in terms of their importance for diverse cell functions. Mitochondria are dynamic structures that undergo both fusion and fission under various conditions, which according to a paper published in Genes and Development now includes activation of the Hippo pathway in Drosophila melanogaster and mammalian cells.

The Hippo pathway regulates cell size and growth, and its deregulation has been implicated in tumour development. Given this, Raghavendra Nagaraj, Shubha Gururaja-Rao and colleagues investigated whether this pathway also impinges on mitochondrial regulation. They looked at cells in D. melanogaster that overexpressed Yorkie (YKI), a transcriptional co-activator downstream of the kinase Hippo, and found the number and size of the mitochondria significantly increased. Mutations in components of the Hippo pathway that result in the activation of this pathway had a similar effect, but overexpression of the microRNA bantam, a transcriptional target of YKI that is known to result in tissue overgrowth, had no effect on mitochondria. Indeed, overexpression of $\mathrm{YKI}$ in post-mitotic cells indicated that the effect of YKI on mitochondria was not linked to cell proliferation. Overexpression of YAP2, a YKI homologue in mammalian cells, also resulted in increased size of mitochondria in four of five human cancer cell lines in vitro.

Investigation of the mitochondrial changes using confocal and electron microscopy indicated that the mitochondria undergo fusion in cells overexpressing YKI or YAP2. These larger mitochondria did not seem to alter the levels of specific metabolites in the cells, but the authors noted a substantial reduction in the generation of reactive oxygen species (ROS) in cells overexpressing YAP2. A genome-wide microarray analysis of transcripts associated with mitochondrial function indicated that two genes involved in mitochondrial fusion, opa1 and mitochondrial assembly regulatory factor (Marf), showed modest upregulation confocal and electron

microscopy indicated that the mitochondria undergo fusion in cells overexpressing YKI or YAP2

in cells overexpressing YKI. Further analyses showed that 261 mitochondrialrelated genes, including opa1 and Marf, were bound by $\mathrm{YKI}$ in association with the DNA-binding factor Scalloped and these included genes involved in the antioxidant pathway and electron transport chain complex 1 , thus potentially explaining the reduction in ROS levels. Genetic loss of opa1 or Marf in D. melanogaster suppressed the mitochondrial fusion phenotype in cells overexpressing $\mathrm{YKI}$, and this correlated with suppressed proliferation.

How the effects of YKI on opa1 and Marf interact with the other known functions of the Hippo pathway awaits investigation.

Nicola McCarthy

Chief Editor, Nature Reviews Cancer

This article is modified from the original in Nature Rev. Cancer (doi:10.1038/nrc3362).

ORIGINAL RESEARCH PAPER Nagaraj, R. et al.

Control of mitochondrial structure and function by the Yorkie/YAP oncogenic pathway. Genes Dev. 27 Aug 2012 (doi:10.1101/gad.183061.111) FURTHER READING Westermann, B.

Mitochondrial fusion and fission for cell life and death. Nature Rev. Mol.Cell. Biol. 11, 872-884 (2010) 